\section{A Guide to the Design and Use of Mismatched and Degenerate Primers}

\author{
Shirley Kwok, Sheng-Yung \\ Chang, John J. Sninsky, \\ and Alice Wang
}

Roche Molecular Systems, Inc., Alameda, California 94501
Although simple in concept, PCR requires a myriad of complex interactions between template, primers, deoxynucleoside triphosphates, and DNA polymerase to successfully accomplish targeted amplification. Primer design in conjunction with changes in the concentrations of reaction components and thermal cycling parameters provides a versatility available in no other molecular technique. For example, PCR can be employed to accommodate mismatches in the primer-template duplex, thereby permitting amplification not only of related sequences but also of uncharacterized sequences. It can also be designed to amplify selectively a small number of mutant genes with single base alterations in a vast background of normal genes.

The flexibility of PCR has greatly simplified molecular manipulations. Procedures for altering a particular template sequence, which in the past required several steps, can now be performed with only a few manipulations. Because 3 ' and internal mismatches between primer-template duplexes are tolerated under appropriate conditions, point mutations and desired restriction endonuclease sites can be directly introduced into the primers. Likewise, nucleotide insertions and deletions can be similarly introduced into the amplified product via the primers.

Degenerate primers have made it possible to amplify related but distinct nucleic acid sequences as well as to amplify targets for which only amino acid sequences are available. The range of possible applications precludes providing detailed protocols for each. Instead, the delineation of the critical parameters and a guide for representative use will be summarized. Depending on the application, a subset of these recommendations or suggestions may suffice.

\section{MISMATCH DISCRIMINATION}

Primers can be tailored to amplify selectively targets that vary by a single nucleotide (for review, see Ugozzoli and Wallace). ${ }^{(1)}$ Genetic diseases commonly arise from single base-pair mutations. Sickle cell anemia, for example, results from an $A$ to $T$ transversion in codon 6 of the human $\beta$-globin sequence. $^{(2)}$ Single point mutations at codons 12,13 , or 61 of the ras genes have resulted in activation of the proto-oncogene that has been associated with a high frequency of human cancers. ${ }^{(3)}$ Resistance to HIV antivirals such as zidovudine, $\mathrm{ddl}, \mathrm{ddC}$, and pyridinone reverse transcriptase inhibitors are conferred by multiple single base mutations. ${ }^{(4-7)}$

The discrimination of different PCR targets is based on the fact that Taq DNA polymerase lacks a $3^{\prime} \rightarrow 5^{\prime}$ exonuclease activity ${ }^{(8)}$ and that mismatched $3^{\prime}$ termini are extended at a lower rate than matched termini. Various mismatches are extended at different efficiencies. ${ }^{(9,10)}$ Multiple acronyms have been used to describe sequence-specific amplification, including ASPCR (allele-specific PCR), ${ }^{(1)}$ ARMS (amplification refractory mutation system), ${ }^{(\overline{1} 1)}$ MAMA (mismatch amplification mutation assay), ${ }^{(12)}$ and PASA (PCR amplification of specific alleles). ${ }^{(13)}$ The appellation allele-specific is somewhat of a misnomer because rare somatic mutations can also be identified using this approach.

In designing primer-pair systems to discriminate mutant from wild type sequences, one needs to first examine the mutations involved. The placement of a mismatch at the 3 ' terminus of a primer-template duplex is more detrimental to PCR than internal mismatches. However, not all 3'-terminal mismatches affect PCR equally. Kwok et al. ${ }^{(14)}$ demonstrated that even in a system designed to tolerate mismatches, 3 '-terminal mismatches involving A:G, G:A, C:C, and G:G reduced product yield by 100 -fold and A:A by 20 -fold, whereas all other mismatches had little effect. Because G:T mismatches are more stable than other mismatches, ${ }^{(15)} \mathrm{G}$ :T should be avoided when design- 
ing primers, although this mismatch has been used successfully. ${ }^{(16)}$ On the other hand, one can still take advantage of this mismatch by targeting the complementary strand that forms an $\mathrm{A}: \mathrm{C}$ mismatch. It is important to note that sequence context can significantly alter the properties of these mismatches, and as much as a 10-fold difference among sites has been reported. ${ }^{(10,17)}$

Occasionally, a single-base mismatch at the $3^{\prime}$ terminus is insufficient to achieve the desired level of discrimination, particularly when the ratio of mutant to wild-type sequence is low. A 3'-terminal mismatch coupled with an additional mismatch either 1,2 , or 3 bases from the $3^{\prime}$ terminus can increase discrimination. ${ }^{(11,12)}$ Therefore, if necessary, the deliberate introduction of a second mutation 1-3 bases from the $3^{\prime}$ terminus will destabilize the $3^{\prime}$ end and provide even greater differentiation. To increase the ratio of mutant to wild-type sequences, an "enriched" PCR was employed by Kahn et al. ${ }^{(18)}$ to identify mutations at codon 12 of the K-ras gene. A restriction enzyme site was generated in the wild-type sequence using a mismatched primer in the first round of amplification. The wild-type sequences were eliminated by digesting the PCR products with the appropriate restriction enzyme, and mutant sequences were enriched with a second round of amplification using mutant-specific primers.

The use of pairs of sequence-specific primers for PCR [also known as double ARMS $^{(19)}$ or double PASA $\left.{ }^{(20)}\right]$ has enabled direct haplotype determination to be achieved in a single amplification. The procedure is particularly attractive for determining haplotypes of individuals who are heterozygous at a number of polymorphic sites and/or in whom pedigree samples are not available. In addition, by carrying out nested PCR, it should be possible to identify regions that contain as many as four cis mutations. Such an application would be valuable for analysis of mutations in the HIV genome that confer zidovudine resistance.

In addition to 3 '-terminal mismatches, other factors should also be considered. Shorter primers $\left(<20\right.$ bases, $\left.T_{m}<55^{\circ} \mathrm{C}\right)$, lower dNTP levels, ${ }^{(21)}$ higher annealing temperatures, lower primer, $\mathrm{MgCl}_{2}$, and enzyme concentrations, and fewer cycles increase the stringency of the amplifications and can be used to skew amplifications to favor the target sequence (see Table 1). Tada et al. ${ }^{(22)}$ recently found that the Stoffel fragment, an amino-terminally truncated variant of Taq DNA polymerase, when used in combination with hot start ${ }^{(23)}$

TABLE 1 Flexibility of PCR Amplification
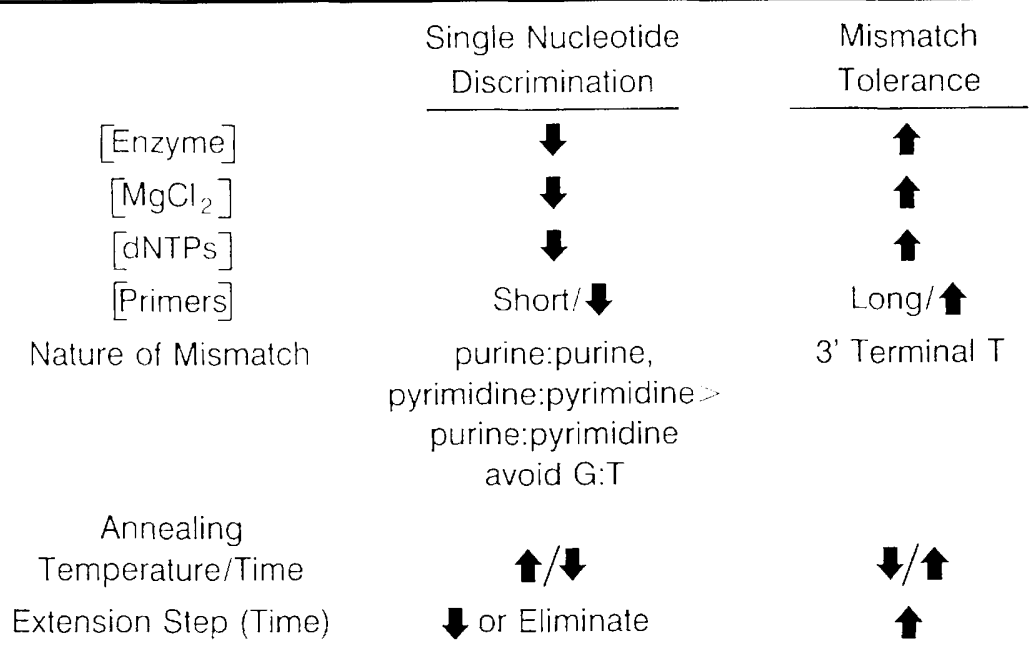

Parameters affecting mismatch discrimination and mismatch tolerance of PCR amplification. Brackets represent the concentration of the parameter. 
further enhanced discrimination. The detection of a small number of single base mutant genes in a $10^{4}$ - to $10^{5}$-fold excess of unaltered genes has been demonstrated successfully in the aforementioned studies.

\section{MISMATCH TOLERANCE}

Although the effects of primer-template mismatches aid in the design of primers for sequence or allele-specific amplification, these effects can also be used to design primers to maximize detection of variant sequences. Most notably, retroviruses and RNA viruses replicate with less fidelity than many DNA viruses and therefore viral genomes within and among patients may vary. False negatives due to mismatches at the $3^{\prime}$ terminus can be minimized by designing primers with a 3 '-terminal $T$. Primers that terminate in $T$ were efficiently extended even when mismatched with $T, G$, or $C .{ }^{(14)}$ Our unpublished studies likewise demonstrate that $3^{\prime}$ terminal dU primers also tolerate mismatches. However, as a rule, $3^{\prime}$-terminal mismatches should be avoided if at all possible.

For amplification of closely related, yet distinct, sequences, chimeric primers can be designed. This approach is particularly attractive when the number of base differences between two sequences in the primer-binding region is small. The primer used, then, is not homologous to either sequence but has a limited number of mismatches to both. By designing the primers so that the mismatches are in the middle or at the $5^{\prime}$ terminus, amplification of both target sequences can occur without compromise. Kwok et al. ${ }^{(24)}$ have described this approach for amplification of HTLV I and II.

The use of primers that are at least 25 bases long $\left(T_{m}>70^{\circ} \mathrm{C}\right)$, high dNTP concentrations $(800 \mu \mathrm{M})$, and annealing temperatures below the $T_{m}$ of the primers will better accommodate mismatches (Table 1). The increase in specificity conferred by hot start PCR may not be suitable if accommodation of mismatches is desired.

Because single-base mismatches can significantly lower the melting temperature of a probe-target duplex, modified bases such as 5-bromodeoxyuracil and 5-methyldeoxycytosine have been used to increase duplex stability. ${ }^{(25)}$ These bases have enabled the use of short oligonucleotides such as the NotI octadeoxynucleotide to probe genomic libraries. ${ }^{(25)}$ The use of 2,6-diaminopurine, an adenine analog, may similarly enhance the stability of duplexes. ${ }^{(26)}$ The stability of these nucleotide duplexes is influenced by base stacking and also by sequence context. One, however, needs to bear in mind the bases incorporated opposite these unconventional bases during PCR.

\section{DEGENERATE PRIMERS}

Some PCR applications require that primers not only tolerate mismatches but also accommodate primer binding to an unknown sequence. Because of the degenerate nature of the genetic code, a given amino acid may be encoded by different triplets. The number of triplets that encode each amino acid is listed in Table 2 . The degenerate primers are designed as a pool of all the possible

TABLE 2 Codon Degeneracy

\begin{tabular}{ll}
\hline Number of codons & Amino acids \\
\hline 1 & Met, Trp \\
2 & Phe, Tyr, His, Gln, Asn, Lys, Asp, Glu, Cys \\
3 & Ile \\
4 & Val, Pro, Thr, Ala, Gly \\
6 & Leu, Ser, Arg \\
\hline
\end{tabular}


combinations of nucleotides that could code for a given amino acid sequence. Degenerate oligonucleotides can either be synthesized individually and then pooled or multiple bases can be programmed at one position in the DNA synthesizer. Because of the differential stability of the blocked bases, care should be taken to use fresh reagents to ensure reproducible and equivalent synthesis of degenerate primers. ${ }^{(27)}$ The degeneracy of a given primer pool can be determined by multiplying the number of possible nucleotides at each position. Mixed oligonucleotide primers derived from an amino acid sequence can be used to amplify a specific sequence from genomic DNA or cDNA that, in turn, can then be used as a probe to screen a genomic/cDNA library for the corresponding clones. ${ }^{(28,29)}$ This is a powerful technique for obtaining a DNA probe when only a limited portion of a protein sequence is available for a sought-after gene. Degenerate primers based on the amino acid sequence of conserved regions were also used to search for members of a gene family, ${ }^{(30)}$ homologous genes from different species, ${ }^{(31)}$ or related viruses. ${ }^{(32-34)}$

When designing degenerate primers, the degeneracy of the genetic code for the selected amino acids of the region targeted for amplification must be examined. Obviously, selection of amino acids with the least degeneracy is desirable because it provides the greatest specificity. Several approaches can be considered for increasing the specificity of the amplification using degenerate primers. First, the pools may be synthesized as subsets such that one pool may contain either a $\mathrm{G}$ or $\mathrm{C}$ at a particular position, whereas the other contains either an $\mathrm{A}$ or $\mathrm{T}$ at the same position. Second, the degeneracy of the mixed primer may be reduced by using the codon bias for translation. ${ }^{(35)}$ Third, the size of the degenerate primers can be as short as 4-6 amino acid codons in length. Six to nine base extensions that contain restriction enzyme sites can be added to the $5^{\prime}$ terminus to facilitate cloning. Mack and Sninsky showed that for short primers, the $5^{\prime}$ extensions facilitated amplification. ${ }^{(26)}$ Fourth, degeneracy at the $3^{\prime}$ end of the primer should be avoided, because single-base mismatches may obviate extension. Finally, the inclusion of deoxyinosine at some ambiguous positions may reduce the complexity of the primer pool. $^{(36,37)}$

Inosine occurs naturally in the wobble position of the anticodon of some transfer RNAs and is known to form base pairs with $A, C$, and $U$ in the translation process. Several experiments have suggested that deoxyinosine might be an "inert" base; its presence in an oligonucleotide sequence seems neither to disturb DNA duplex formation nor to destabilize the duplex. ${ }^{(36,38)}$ Independent of sequence effects, the order of stabilities of base-pairing is $\mathrm{I}: \mathrm{C}>>\mathrm{I}: \mathrm{A}>\mathrm{I}: \mathrm{T}=\mathrm{I}: \mathrm{G} \cdot{ }^{(38)}$ The deoxyinosine-containing primers have been used successfully to amplify cDNA or genomic fragments for the generation of DNA probes from a peptide sequence. ${ }^{(39,40)}$ Deoxyinosine was generally incorporated at base positions with three- to fourfold redundancy. This method may be useful for proteins with highly degenerate codons. Inosine usually directs the incorporation of $C$ when in the template. Unfortunately, the studies to date do not discern whether inosine actually assists mismatched priming. Another approach pioneered by Brown and colleague ${ }^{(41)}$ exploits the use of unconventional bases that are capable of base-pairing with either a purine or a pyrimidine.

It has been observed that the PCR thermal profile can dramatically alter the success rate of degenerate PCR. ${ }^{(42)}$ The preferred degenerate PCR amplification profile starts with a nonstringent annealing temperature $\left(35-45^{\circ} \mathrm{C}\right)$ for $2-5$ cycles, followed by $25-40$ cycles at a more stringent annealing temperature. The relaxed annealing conditions allow the short complementary primer portion to hybridize to the target. After the second cycle of amplification, the 5' extension becomes incorporated into the amplified product and will serve as the template for subsequent rounds of amplification. By shifting 
to a more stringent annealing temperature, increased specificity can be achieved. Furthermore, a 4 - to 5-min ramp time between the annealing and extension temperatures may also result in greater specificity.

\section{MUTAGENESIS PRIMERS}

With the advent of PCR, recombinant DNA procedures such as M13 phage site-directed mutagenesis and the construction of "fusion proteins" have been greatly simplified. ${ }^{(43,44)}$ There are several advantages of generating a mutation with mismatched primers by PCR. First, PCR provides substantial flexibility in the types of mutations that can be introduced. For example, single-base substitutions, deletions, and insertions of different sizes can all be easily generated with one or two rounds of PCR. Second, the mutation(s) of interest located in the PCR product can be cloned into any desirable vector by a single cloning step. Third, almost all the clones harbor the expected mutation.

Conventional methods for construction of sequences encoding a fusion protein require several manipulations. In addition, the sequence at the junction is generally not ideal because the restriction enzyme selected for the joining of the two sequences is usually not present in the native sequence. Therefore, it is difficult to construct a sequence encoding an authentic fusion protein. With PCR, the junction of two segments can easily be engineered into the primers, thereby eliminating extraneous bases.

The location of the mutated base(s) in the primers depends on the nature of the desired mutation. To introduce single-base substitutions and insertions at a location with a unique restriction enzyme site nearby, only two primers are needed (Fig. 1A). A mismatched mutagenesis primer (P1) with the desired mutation sequence and restriction enzyme site sequence (site $X)$ is paired with another primer (P2) with the second restriction enzyme site (site Y) for PCR. The mismatch(es) should be placed in the middle of a 24- to 36-base oligonucleotide. The PCR product can be digested with enzymes $X$ and $Y$ and cloned into a suitable vector. For generating mutations in a sequence that does not contain a convenient restriction enzyme site nearby, two sets of primers are needed for two rounds of PCR (Fig. 1B). Primer 1 (P1) paired with mutagenesis primer $\mathrm{P} 2$ and mutagenesis primer 3 (complementary to primer P2) paired with primer 4 (P4) are used in two separate PCR reactions in the first round of PCR. Following the first round of PCR, a small aliquot ( $1 / 20$ to $1 / 50$ ) of the two PCR products (or gel-purified PCR products) are mixed and amplified in the presence of primers 1 and 4 in the second round of PCR. Because the two PCR products from the first round of PCR have overlapping sequences at the "mutagenized end", one of the two partially matched duplexes formed after denaturation and annealing will serve as the template in the second round of PCR. The PCR product from the second round of amplification is used for cloning.

The introduction of large deletions and the fusion of sequences can be accomplished in a similar manner. The mutagenesis primers for generating a large deletion should contain sequences flanking the deletion region (Fig. 1C). Both mutagenesis primers ( $\mathrm{P} 2$ and $\mathrm{P} 3$ ) should have a region of $>12$ bases at the 3 ' terminus that matches the target sequence and a mismatched tail of $>7$ bases that matches the region flanking the other end of the deletion. The length of the overlapping sequences at the mutagenized end of two firstround PCR products is determined by the total number of bases in the tails of primers $\mathrm{P} 2$ and $\mathrm{P} 3$. For the joining of two target sequences, the length of the mismatched tail of mutagenesis primers should be $>12$ bases (Fig. 1D). Primers 1 and 4 illustrated in Figure 1, B-D, are in the regions with restriction enzyme sites for cloning of the PCR product. Alternatively, the restriction 
A

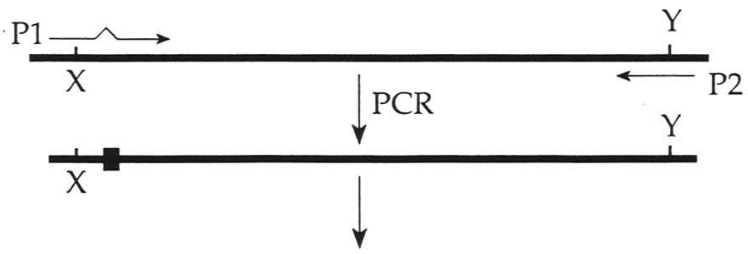

digest with enzymes $X$ and $Y$

B
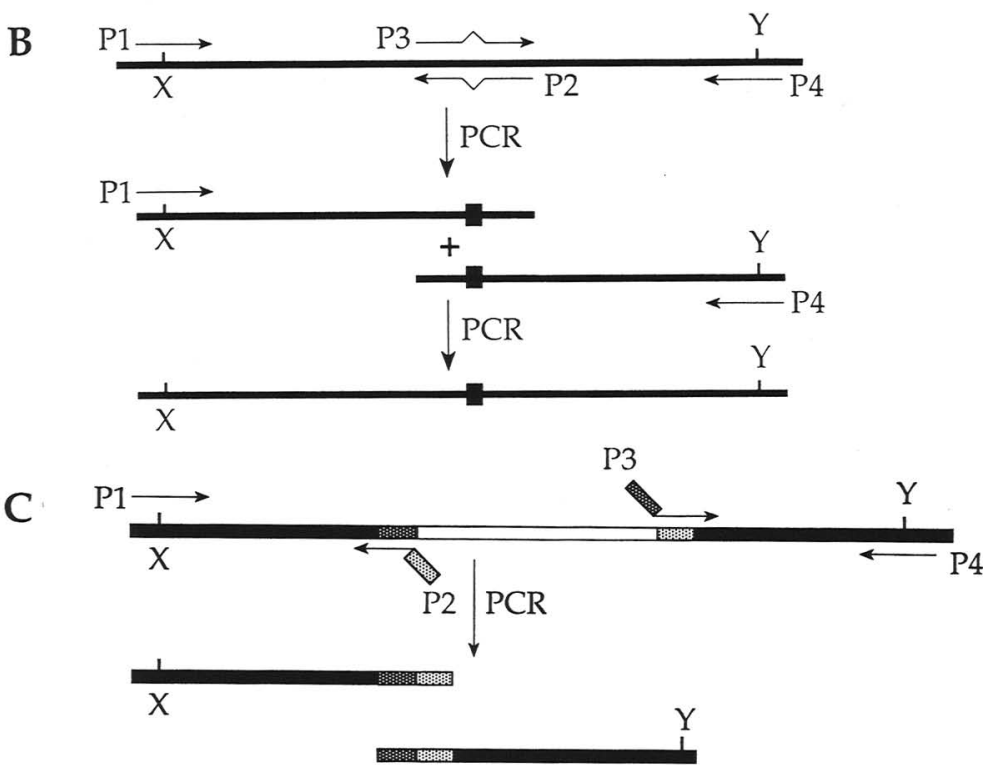

Target A

Target B

D
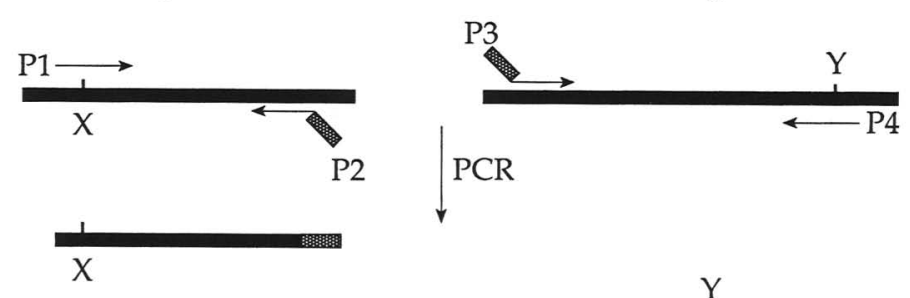

Y

FIGURE 1 Strategies of using mismatched primers for generating mutations $(A-C)$, and fusion of two fragments of DNA $(D)$. Bends in arrows represent the mismatched sequence between primer and target. ( $\square$ ) The mutation introduced by the mismatched primer. (X and Y) Two restriction enzyme sequences. The shaded areas with the same pattern have identical sequence.

enzyme site for cloning can be introduced with the primer containing the sequence of the restriction site and a few extra bases to ensure enzyme digestion at the $5^{\prime}$ terminus.

The length of the primer used in these experiments depends on the G/C content of the sequence and the type of desired mutation. In general, 12-14 bases of perfect complementarity $\left(T_{m} \sim 40^{\circ} \mathrm{C}\right)$ at the $3^{\prime}$ terminus are sufficient for a primer to be annealed and extended, particularly if it is not especially A-T rich. The annealing temperature can also be controlled to achieve an efficient amplification.

If the nucleotide sequence of the mutated fragment is important for further analysis, the location of primer 2 in Figure 1A and primer pair P1/P4 in Figure 1, B-D, should as close as possible to the mutagenesis primer(s) to minimize the number of nucleotides to be confirmed by sequencing. The conditions to minimize the misincorporation of Taq DNA polymerase should be observed. The parameters of the PCR reaction, such as the concentration 
of dNTP and $\mathrm{MgCl}_{2}$, and $\mathrm{pH}$, all have effects on the fidelity of the enzyme. ${ }^{(45)}$ The amount of template and the number of cycles of amplification should be properly controlled to minimize amplification at the plateau phase. Other proofreading DNA polymerases, such as those from Thermotoga maritima (UlTma), Thermococcus litoralis [(Vent)], and Pyococcus furiosus (Pfu), can be used as alternative enzymes for mutagenesis PCR. However, one should bear in mind that the proofreading activity of these enzymes also degrades primers, single-stranded PCR products, and template; therefore, their use may require careful optimization.

\section{CONCLUSION}

Although the published procedures noted were successful, very few comprehensive studies have been performed to understand fully the precise mechanisms involved. As a result, the amount of information available is perhaps less than what might have been expected given the large number of studies carried out. The procedures outlined above are intended to serve as guidelines for the design and use of mismatched and degenerate primers. Depending on the particular PCR application, a subset of these recommendations or suggestions may suffice. Familiarity with the accomplishments and limits of published studies, an understanding of the principles of enzymology and nucleic acid hybridization, and a willingness to reflect first on the goal before embarking on experimentation will increase an investigator's likelihood of success. PCR - like any good tool - can be productive with minimal familiarity. However, understanding the intricacies of how it works and the breadth of potential alterations permits greater exploration of its limits and its applications to new areas.

\section{ACKNOWLEDGMENTS}

We thank Shari Kurita for graphics and JoAnn Williams for manuscript preparation. We thank David Gelfand for reviewing the manuscript. It is impossible to cite or acknowledge the large number of individuals both at Roche Molecular Systems and elsewhere who have contributed to the studies described in this manuscript. We thank all of them for their efforts.

\section{REFERENCES}

1. Ugozzoli, L. and R.B. Wallace. 1991. Allele-specific polymerase chain reaction. Methods: $A$ companion to Methods in Enzymol. 2: 42-48.

2. Wu, D.Y., L. Ugozzoli, B.K. Pal, and R.B. Wallace. 1989. Allele-specific enzymatic amplification of $\beta$-globin genomic DNA for diagnosis of sickle cell anemia. Proc. Natl. Acad. Sci. 36: $2757-2760$.

3. Bos, J.L. 1989. Ras oncogens in human cancer: A review. Cancer Res. 49: 4682-4689.

4. Larder, B.A, P. Kellan, and S.D. Kemp. 1991. Zidovudine resistance predicted by direct detection of mutations in DNA from HIV-infected lymphocytes. AIDS 5: 137-144.

5. St. Clair, M.H., J.L. Martin, G. Tudor-Williams, M.C. Bach, C.L. Vavro, D.M. King, P. Kellam, S.D. Kemp, and B.A. Larder. 1991. Resistance to ddI and sensitivity to AZT induced by a mutation in HIV-1 reverse transcriptase. Science 253: 1557-1559.

6. Nunberg, J.H., W.A. Schleif, E.J. Boots, J.A. O'Brien, J.C. Quintero, J.M. Hoffman, E.A. Emini, and M.E. Goldman. 1991. Viral resistance to human immunodeficiency virus type 1-specific pyridinone reverse transcriptase inhibitors. J. Virol. 65: 4887-4892.

7. Larder, B.A. and C.A.B. Boucher. 1993. PCR detection of human immunodeficiency virus drug resistance mutations. In Diagnostic molecular biology (ed. D.H. Persing, T.F. Smith, F.C. Tenover, and T.J. White), pp. 527-533. American Society for Microbiology, Washington D.C.

8. Lawyer, F.C., S. Stoffel, R.K. Saiki, K. Myambo, R. Drummond, and D.H. Gelfand. 1989. Isolation characterization, and expression in Escherichia coli of the DNA polymerase gene from Thermus aquaticus. J. Biol. Chem. 264: 6427-6436. 
9. Creighton, S., M. Huang, H. Cai, N. Arnheim, and M.F. Goodman. 1992. Base mispair extension kinetics: Binding of avian myelobalstosis reverse transcriptase to matched and mismatched base pair termini. J. Biol. Chem. 267: 2633-2639.

10. Huang, M., N. Arnheim, and M.F. Goodman. 1992. Extension of base mispairs by Taq DNA polymerase: Implications for single nucleotide discrimination in PCR. Nucleic Acids Res. 20: $4567-4573$.

11. Newton, C.R., A. Graham, L.E. Heptinstall, S.J. Powell, C. Summers, N. Kalsheker, J.C. Smith, and A.F. Markham. 1989. Analysis of any point mutation in DNA. The amplification refractory mutation system (ARMS). Nucleic Acids Res. 17: 2503-2516.

12. Cha, R.S., H. Zarbl, P. Keohavong, and W.G. Thilly. 1992. Mismatch amplification mutation assay (MAMA): Application to the c-H-ras gene. PCR Methods Applic. 2: 14-20.

13. Dutton, C. and S.S. Sommer. 1991. Simultaneous detection of multiple single-base alleles at a polymorphic site. BioTechniques 11: 700-702.

14. Kwok, S., D.E. Kellogg, N. McKinney, D. Spasic, L. Goda, and J.J. Sninsky. 1990. Effects of primer-template mismatches on the polymerase chain reaction: Human immunodeficiency virus type 1 model studies. Nucleic Acids Res. 18: 999-1005.

15. Kidd, V.J., R.B. Wallace, K. Itakura, and S.L.C. Woo. 1983. $\alpha_{1}$-Antitrypsin deficiency detection by direct analysis of the mutation in the gene. Nature 304: 230-234.

16. Li, H., X. Cui, and N. Amheim. 1990. Direct electrophoretic detection of the allelic state of single DNA molecules in human sperm by using the polymerase chain reaction. Proc. Natl. Acad. Sci. 87: 4580-4584.

17. Mendelman, L.V., M.S. Boosalis, J. Petruska, and M.F. Goodman. 1989. Nearest neighbor influences on DNA polymerase insertion fidelity. J. Biochem. 264: 14415-14423.

18. Kahn, S.M., W. Jiang, T.A. Culbertson, l.B. Weistein, G.M. Williams, N. Tomita, and Z. Ronai. 1991. Rapid and sensitive nonradioactive detection of mutant K-ras genes via "enriched" PCR amplification. Oncogene 6: 1079-1083.

19. Lo, Y-M.D., P. Patel, C.R. Newton, A.F. Markham, K.A. Fleming, and J.S. Wainscoat. 1991. Direct haplotype determination by double ARMS: Specificity, sensitivity and genetic applications. Nucleic Acids Res. 19: 3561-3567.

20. Sarkar, G. and S.S. Sommer. 1991. Haplotyping by double PCR amplification of specific alleles, BioTechniques 10: 436-440.

21. Ehlen, T. and L. Dubeau. 1989. Detection of ras point mutations by polymerase chain reaction using mutation-specific, inosine-containing oligonucleotide primers. Biochem Biophys. Res. Commun. 160: 441-447.

22. Tada, M., M. Omata, S. Kawai, H. Saisho, M. Ohto, R.K. Saiki, and J.J. Sninsky. 1993. Detection of ras gene mutations in pancreatic juice and peripheral blood of patients with pancreatic adenocarcinoma. Cancer Res. 53: 1-3.

23. Chou, Q., M. Russell, D.E. Birch, J. Raymond, and W. Bloch. 1992. Prevention of pre-PCR mis-priming and primer dimerization improves low-copy-number amplifications. Nucleic Acids. Res. 20: $1717-1723$.

24. Kwok, S., D. Kellogg, G. Ehrlich, B. Poiesz, B. Bhagavati, and J.J. Sninsky. 1988. Characterization of a sequence of human T cell leukemia virus type I from a patient with chronic progressive myelopathy. J. Inf. Dis. 158: 1193-1197.

25. Hoheisel, J.D., A.G. Craig, and H. Lehrach. 1990. Effect of 5-bromo- and 5-methyldeoxycytosine on duplex stability and discrimination of the NotI octadeoxynucleotide. J. Biol. Chem. 265: $16656-16660$.

26. Cheong, C., I. Tinoco, A. Chollet. 1988. Thermodynamic studies of base pairing involving 2,6-diaminopurine. Nucleic Acids Res. 16: 5115-5122.

27. Bartel, D.P. and J.W. Szostak. 1993. Isolation of new ribozymes from a large pool of random sequences. Science 261: 1411-1418.

28. Lee, C.C., X. Wu, R.A. Gibbs, R.G. Cook, D.M. Muzny, and C.T. Caskey. 1988. Generation of cDNA probes directed by amino acid sequence: Cloning of urate oxidase. Science 239: 12881291.

29. Girgis, S.I., M. Slevizaki, P. Denny, G.J.M. Ferrier, and S. Legon. 1988. Generation of DNA probes for peptides with highly degenerate codons using mixed primer PCR. Nucleic Acids Res. 16: 10371.

30. Wilks, F.F., R.R. Kurban, C.M. Hovens, and S.J. Ralph. 1989. The application of the polymerase chain reaction to cloning members of the protein tyrosine kinase family. Gene 85: $67-74$.

31. Kopin, A.S., M.B. Wheeler, and A.B. Leiter. 1990. Secretin: Structure of the precursor and tissue distribution of the mRNA. Proc. Natl. Acad. Sci. 87: 2299-2303.

32. Mack, D. and J.J. Sninsky. 1988. A sensitive method for the identification of uncharacterized viruses related to known virus groups: Hepadnavirus model system. Proc. Natl. Acad. Sci. 85: $6977-6981$

33. Manos, M.M., Y. Ting, D.K. Wright, A.J. Lewis, T.R. Broker, and S.M. Wolinsky. 1989. Use of polymerase chain reaction amplification for the detection of genital human papillomaviruses. Cancer Cells (Molecular diagnostics of human cancer) 7: 209-214. 
34. Shih, A., R. Misra, and M.G. Rush. 1989. Detection of multiple, novel reverse transcriptase coding sequences in human nucleic acids: Relation to primate retroviruses. J. Virol. 63: 64 75.

35. Wada, K., Y. Wada, H. Doi, F. Ishibashi, G. Takashi, and T. Ikemura. 1991. Codon usage tabulated from the GenBank genetic sequence data. Nucleic Acids Res. 19: 1981.

36. Ohtsuka, E., S. Matsuka, M. Ikehara, Y. Takahashi, and K. Matsubara. 1985. An alternative approach to deoxyoligonucleotides as hybridization probes by insertion of deoxyinosine at ambiguous codon positions. J. Biol. Chem. 260: 2605-2608.

37. Sakanari, J.A., C.E. Staunton, A.E. Eakin, and C.S. Craik. 1989. Serine proteases from nematode and protozoan parasites: Isolation of sequence homologs using generic molecular probes. Proc. Natl. Acad. Sci. 86: 4863-4867.

38. Martin, F.H., M.M. Castro, F. Aboul-ela, and I. Tinoco, Jr. 1985. Base pairing involving deoxyinosine: Implication for probe design. Nucleic Acids Res. 13: 8927-8938.

39. Schuchman, E.H., C.E. Jackson, and R. Desnick. 1990. Human arylsufatase B: MOPAC Cloning, nucleotide sequence of a full-length CDNA, and regions of amino acid identity with arylsufatases A and C. Genomics 6: 149-158.

40. Aarts, J.M.M.J.G., J.G.J. Hontelez, P. Fischer, R. Verkerk, A. van Kamen, and P. Zabel. 1991. Acid phosphatase-1, a tightly linked molecular marker for root-knot nematode resistance in tomato: From protein to gene, using PCR and degenerate primers containing deoxyinosine. Plant Mol. Biol. 16: 647-661.

41. Lin, P.K.T. and D.M. Brown. 1992. Synthesis of oligodeoxyribonucleotides containing degenerate bases and their use as primers in the polymerase chain reaction. Nucleic Acids Res. 20: $5149-5152$.

42. Compton, T. 1990. Degenerate primers for DNA amplification. PCR Protocols: A guide to methods and applications (ed. M.A. Innis, D.H. Gelfand, J.J. Sninsky, and T.J. White) pp. 39-45. Academic Press, San Diego, CA.

43. Scharf, S.J. 1990. Cloning with PCR. In PCR Protocols: A guide to methods and applications (ed. M.A. Innis, D.H. Gelfand, J.J. Sninsky, and T. White), pp. 84-91. Academic Press, San Diego, CA.

44. Higuchi, R. 1990. Recombinant PCR. In PCR Protocols: A guide to methods and applications (ed. M.A. Innis, D.H. Gefland, J.J. Sninsky, and T. White) pp. 177-183. Academic Press, San Diego, CA.

45. Eckert, K.A. and T.A. Kunkel. 1990. High fidelity DNA syntheseis by Thermus aquaticus DNA polymerase. Nucleic Acids Res. 18: 3739-3744. 


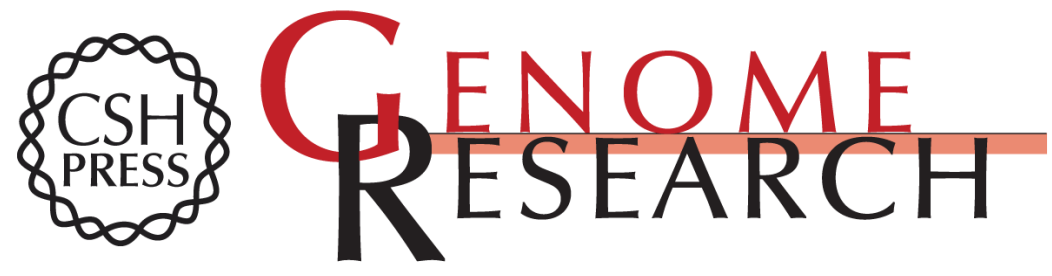

\section{A guide to the design and use of mismatched and degenerate primers.}

S Kwok, S Y Chang, J J Sninsky, et al.

Genome Res. 1994 3: S39-S47

References This article cites 41 articles, 15 of which can be accessed free at: http://genome.cshlp.org/content/3/4/S39.full.html\#ref-list-1

License

Email Alerting

Receive free email alerts when new articles cite this article - sign up in the box at the Service top right corner of the article or click here.

\section{Affordable, Accurate Sequencing.}

To subscribe to Genome Research go to: https://genome.cshlp.org/subscriptions 